\title{
Pragmatic Recommendations for the Management of Acute Respiratory Failure and Mechanical Ventilation in Patients with COVID-19 in Low- and Middle-Income Countries
}

\author{
Ary Serpa Neto, ${ }^{1,2,3,4 \star}$ William Checkley, ${ }^{5,6}$ Chaisith Sivakorn, ${ }^{7}$ Madiha Hashmi, ${ }^{8}$ Alfred Papali, ${ }^{9}$ and Marcus J. Schultz ${ }^{3,10,11}$ for the \\ COVID-LMIC Task Force and the Mahidol-Oxford Research Unit (MORU) \\ ${ }^{1}$ Australian and New Zealand Intensive Care Research Centre, Monash University, Melbourne, Australia; ${ }^{2}$ Department of Critical Care Medicine, \\ Hospital Israelita Albert Einstein, São Paulo, Brazil; ${ }^{3}$ Department of Intensive Care, Amsterdam University Medical Centers, Location 'Academic \\ Medical Center', Amsterdam, The Netherlands; ${ }^{4}$ Data Analytics Research and Evaluation (DARE) Centre, Austin Hospital, University of Melbourne, \\ Melbourne, Australia; ${ }^{5}$ Division of Pulmonary and Critical Care, Department of Medicine, School of Medicine, Johns Hopkins University, Baltimore, \\ Maryland; ${ }^{6}$ Center for Global Non-Communicable Disease Research and Training, School of Medicine, Johns Hopkins University, Baltimore, \\ Maryland; ${ }^{7}$ Department of Clinical Tropical Medicine, Mahidol University, Bangkok, Thailand; ${ }^{8}$ Department of Anaesthesiology, Ziauddin \\ University, Karachi, Pakistan; ${ }^{9}$ Division of Pulmonary and Critical Care Medicine, Atrium Health, Charlotte, North Carolina; ${ }^{10}$ Mahidol-Oxford \\ Tropical Medicine Research Unit (MORU), Mahidol University, Bangkok, Thailand; ${ }^{11}$ Nuffield Department of Medicine, Oxford University, Oxford, \\ United Kingdom
}

\begin{abstract}
Management of patients with severe or critical COVID-19 is mainly modeled after care for patients with severe pneumonia or acute respiratory distress syndrome (ARDS) from other causes, and these recommendations are based on evidence that often originates from investigations in resource-rich intensive care units located in high-income countries. Often, it is impractical to apply these recommendations to resource-restricted settings, particularly in low- and middle-income countries (LMICs). We report on a set of pragmatic recommendations for acute respiratory failure and mechanical ventilation management in patients with severe/critical COVID-19 in LMICs. We suggest starting supplementary oxygen when $\mathrm{SpO}_{2}$ is persistently lower than $94 \%$. We recommend supplemental oxygen to keep $\mathrm{SpO}_{2}$ at 88-95\% and suggest higher targets in settings where continuous pulse oximetry is not available but intermittent pulse oximetry is. We suggest a trial of awake prone positioning in patients who remain hypoxemic; however, this requires close monitoring, and clear failure and escalation criteria. In places with an adequate number and trained staff, the strategy seems safe. We recommend to intubate based on signs of respiratory distress more than on refractory hypoxemia alone, and we recommend close monitoring for respiratory worsening and early intubation if worsening occurs. We recommend low-tidal volume ventilation combined with $\mathrm{FiO}_{2}$ and positive end-expiratory pressure (PEEP) management based on a high $\mathrm{FiO}_{2} /$ low PEEP table. We recommend against using routine recruitment maneuvers, unless as a rescue therapy in refractory hypoxemia, and we recommend using prone positioning for $12-16$ hours in case of refractory hypoxemia $\left(\mathrm{PaO}_{2} /\right.$ $\mathrm{FiO}_{2}<150 \mathrm{mmHg}, \mathrm{FiO}_{2} \geq 0.6$ and PEEP $\geq 10 \mathrm{cmH}_{2} \mathrm{O}$ ) in intubated patients as standard in ARDS patients. We also recommend against sharing one ventilator for multiple patients. We recommend daily assessments for readiness for weaning by a low-level pressure support and recommend against using a T-piece trial because of aerosolization risk.
\end{abstract}

\section{INTRODUCTION}

Management of patients with severe or critical COVID-19 is mainly based on care for patients with severe pneumonia or acute respiratory distress syndrome (ARDS) from other causes, although some aspects of this new disease may demand a different approach. Recommendations for treatment of severe pneumonia and ARDS management have been gathered mainly from investigations in resource-rich intensive care units (ICUs), mostly located in high-income countries (HICs).

It may not be practical to apply these recommendations to resource-restricted settings, particularly in low- and middleincome countries (LMICs). Indeed, high-dependency units and ICUs in LMICs are frequently restricted to availability of infrastructure, equipment, medications, skilled, nurses and doctors. An international task force comprising members from LMICs and HICs, all with direct experience in various LMIC settings, critically appraised a list of questions regarding acute respiratory failure and mechanical ventilation for patients with severe/critical COVID-19.

We provide a list of recommendations and suggestions after pragmatic, experience-based appraisal. A summary of the recommendations is shown in Table 1.

\footnotetext{
${ }^{*}$ Address correspondence to Ary Serpa Neto, Australian and New Zealand Intensive Care Research Centre, Monash University, Level 3, 553 St. Kilda Rd., Melbourne VIC 3004, Australia. E-mail: ary.serpaneto@monash.edu
}

\section{METHODS}

A full description of the methods is provided in the Appendix. In brief, we formulated a set of clearly defined questions regarding acute respiratory failure and mechanical ventilation for patients with suspected or confirmed severe/critical COVID-19 in LMICs. The list of questions was reviewed for content and clarity by other members of the COVID-LMIC Task Force, the full membership of which can be found in the Appendix. After approval, the subgroup assigned one member to search the literature for evidence to answer each question. The literature search was performed in a minimum of one general database (i.e., MEDLINE and Embase) and the Cochrane libraries. Furthermore, we identified investigations from LMICs and searched for unpublished study results. We selected relevant publications, appraised the evidence, and classified the quality of evidence as high, moderate, low, or very low. Recommendations were rated as strong or weak, depending on the quality of evidence and several other factors such as availability, affordability, and feasibility in LMICs. A strong recommendation was worded as "we recommend..." and a weak recommendation as "we suggest. ..," followed by the quality of evidence. A number of recommendations could remain "ungraded," when, in the opinion of the subgroup members, such recommendations were not conducive for the process described previously (Appendix Table 2). The recommendations were reviewed by the subgroup in an iterative process and were later reviewed by the entire Task Force in two rounds. 
TABLE 1

Recommendations and suggestions on acute respiratory failure and mechanical ventilation in COVID-19 patients in low- and middle-income countries (with grading)

\begin{tabular}{|c|c|c|c|}
\hline & & Actual recommendations & Surviving sepsis campaign COVID-19 guideline \\
\hline 1 & Start oxygen & $\begin{array}{l}\text { We suggest starting supplementary } \\
\text { oxygen when } \mathrm{SpO}_{2} \text { is persistently lower } \\
\text { than } 94 \% \text { (weak recommendation, low } \\
\text { quality of evidence) }\end{array}$ & $\begin{array}{l}\text { In adults with COVID-19, we suggest } \\
\text { starting supplemental oxygen if the } \\
\text { peripheral oxygen saturation }\left(\mathrm{SpO}_{2}\right) \text { is } \\
92 \% \text { (weak recommendation, low- } \\
\text { quality evidence) and recommend } \\
\text { starting supplemental oxygen if } \mathrm{SpO}_{2} \text { is } \\
90 \% \text { (strong recommendation, } \\
\text { moderate-quality evidence) }\end{array}$ \\
\hline 2 & Supplement oxygen & $\begin{array}{l}\text { We recommend supplemental oxygen to } \\
\text { keep } \mathrm{SpO}_{2} \text { at } 88-95 \% \text { (strong } \\
\text { recommendation, high quality of } \\
\text { evidence) }\end{array}$ & $\begin{array}{l}\text { In adults with COVID-19 and acute } \\
\text { hypoxemic respiratory failure on } \\
\text { oxygen, we recommend that } \mathrm{SpO}_{2} \text { be } \\
\text { maintained no higher than } 96 \% \text { (strong } \\
\text { recommendation, moderate-quality } \\
\text { evidence) }\end{array}$ \\
\hline 3 & Supplement oxygen & We suggest higher targets for $\mathrm{SpO}_{2}$ in & Not discussed \\
\hline
\end{tabular}

$4 \quad$ Supplement oxygen

5

Supplement oxygen

6

Fever and symptom control

Awake prone positioning

8

Prone positioning

9

Intubation

10

Intubation

11

Intubation settings where continuous pulse oximetry is not available $\left(\mathrm{SpO}_{2}\right.$ at 95-97\%) (weak recommendation, low quality of evidence)

We recommend using low-flow oxygen systems, for example, nasal prongs, a sponge-tipped catheter, or a facial mask for supplementary oxygen, and, where available, a non-rebreathing mask (strong recommendation, low quality of evidence)

We suggest using of HFNO or NIV with monitoring and isolation considerations (especially for NIV) in patients who remain hypoxemic, despite using low-flow oxygen therapy (weak recommendation, low quality of evidence)

We suggest fever control with paracetamol, using cough suppressants in case of severe coughing, and light sedation (weak recommendation, low quality of evidence)

We suggest a trial of awake prone positioning in patients who remain hypoxemic despite use of oxygen, HFNO, or NIV, and who do not exhibit clear signs of respiratory distress; this however requires close monitoring and clear failure and escalation criteria (weak recommendation, low quality of evidence)

We recommend using prone positioning for 12-16 hours in case of refractory hypoxemia $\left(\mathrm{PaO}_{2} / \mathrm{FiO}_{2}<150 \mathrm{mmHg}\right.$, $\mathrm{FiO}_{2} \geq 0.6$, PEEP $\geq 10 \mathrm{cmH}_{2} \mathrm{O}$ ) (strong recommendation, low quality of evidence)

We recommend intubating and initiating invasive ventilation based on signs of fatigue and respiratory distress, rather than refractory hypoxemia alone (weak recommendation, low quality of evidence)

We recommend close monitoring for respiratory worsening and early intubation if worsening occurs (strong recommendation, low quality of evidence)

We recommend staff members to be readily available to closely monitoring a
Not discussed

For adults with COVID-19 and acute hypoxemic respiratory failure despite conventional oxygen therapy, we suggest using HFNC over conventional oxygen therapy (weak recommendation, low-quality evidence)

For critically ill adults with COVID-19 who develop fever, we suggest using acetaminophen/paracetamol for temperature control over no treatment (weak recommendation, low-quality evidence)

Not discussed

For mechanically ventilated adults with COVID-19 and moderate to severe ARDS, we suggest prone ventilation for 12-16 hours over no prone ventilation (weak recommendation, low-quality evidence)

In adults with COVID-19 receiving NIPPV or HFNC, we recommend close monitoring for worsening of respiratory status and early intubation in a controlled setting if worsening occurs (best practice statement)

In adults with COVID-19 receiving NIPPV or HFNC, we recommend close monitoring for worsening of respiratory status and early intubation in a controlled setting if worsening occurs (best practice statement)

Not discussed 


\begin{tabular}{|c|c|c|}
\hline & & Actual recommendations \\
\hline & & $\begin{array}{l}\text { patient when an early intubation was } \\
\text { not performed, and staff should be } \\
\text { trained in timely recognition of } \\
\text { respiratory distress and sign of } \\
\text { worsening of respiratory distress (weak } \\
\text { recommendation, low quality of } \\
\text { evidence) }\end{array}$ \\
\hline 12 & Mechanical ventilation & $\begin{array}{l}\text { We recommend using a low tidal volume } \\
(4-8 \mathrm{~mL} / \mathrm{kg} \text { of } \mathrm{PBM} \text { ), and whenever } \\
\text { possible, a tidal volume } \leq 6 \mathrm{~mL} / \mathrm{kg} \text { of } \\
\text { PBW should be pursued (strong } \\
\text { recommendation, high quality of } \\
\text { evidence) }\end{array}$ \\
\hline 13 & Mechanical ventilation & $\begin{array}{l}\text { We recommend using a "low PEEP/high } \\
\mathrm{FiO}_{2} \text { table" (moderate } \\
\text { recommendation, low quality of } \\
\text { evidence) }\end{array}$ \\
\hline
\end{tabular}
recruitment maneuvers, unless as a rescue therapy in refractory hypoxemia (moderate recommendation, low quality of evidence) recommendation, low quality of evidence) (4-8 $\mathrm{mL} / \mathrm{kg}$ of PBM), and whenever PBW should be pursued (strong recommendation, high quality of e recommend using a "low PEEP/high recommendation, low quality of evidence)

We recommend against sharing one ventilator for multiple patients (strong recommendation, low quality of evidence)

$16 \quad$ Weaning

We recommend daily assessments for readiness for weaning by a low-level pressure support (strong recommendation, low quality of evidence)

$17 \quad$ Weaning

We recommend against using T-piece trials because of contamination risk (weak recommendation, low quality of evidence)

$18 \quad$ Extubation
We recommend using familiar, local extubation protocols to minimize infection risk among healthcare workers (weak recommendation, low quality of evidence)
In mechanically ventilated adults with COVID-19 and ARDS, we recommend using low-tidal volume ventilation $(\mathrm{Vt}$ 4-8 $\mathrm{mL} / \mathrm{kg}$ of PBW), over higher tidal volumes $(\mathrm{Vt}>8 \mathrm{~mL} / \mathrm{kg}$ ) (strong recommendation, moderate-quality evidence)

For mechanically ventilated adults with COVID-19 and moderate to severe ARDS, we suggest using a higher PEEP strategy over a lower PEEP strategy (weak recommendation, low-quality evidence)

For mechanically ventilated adults with COVID-19 and hypoxemia despite optimizing ventilation, we suggest using recruitment maneuvers over not using recruitment maneuvers (weak recommendation, low-quality evidence)

Not discussed

Not discussed

Not discussed

Not discussed

EEP = positive end-expiratory pressure; PWP = predicted body weight; $\mathrm{SpO}_{2}=$ pulse oximetry. Grading: see Appendix for $\mathrm{HFNO}=$ high-1
explanations.

\section{QUESTIONS}

We formulated five clearly defined questions regarding "acute respiratory failure and mechanical ventilation":

1. How to supplement oxygen in COVID-19 patients in LMICs?

2. Should prone positioning be used in COVID-19 in LMICs?

3. When to intubate for invasive ventilation in COVID-19 patients in LMICs?

4. How to set the ventilator in COVID-19 patients in LMICs?

5. How to wean a COVID-19 patient from the ventilator in LMICs?

How to supplement oxygen in COVID-19 patients in LMICs? Background. Supplementary oxygen is a first and essential therapeutic step in the care of hypoxemic COVID-19 patients and may be the dominant focus of care for these patients in LMICs.

Search results. We searched the databases of PubMed and Embase through Ovid until November 1, 2020. The following terms were used, either as medical subject headings (MeSH) terms or as free text words: "COVID-19," "coronavirus," "novel corona," "SARS-CoV-2" and "oxygen," "pneumonia," "oxygen target," "liberal," "conventional" in oxygen initiation and targets; "oxygen therapy," "aerosol," "high-flow nasal oxygen (HFNO)," "non-invasive ventilation (NIV)" in oxygen delivery interfaces; "fever control," "cough suppressants," "sedation" in fever control, use of cough suppressants in case of severe coughing, and light sedation; and "intubation criteria," "hypoxemia," "silent hypoxemia" in initiation of invasive ventilation. From the search, it was found five randomized control trials, six systematic reviews, four narrative reviews, and one observational study, and none of the studies were from LMICs.

Evidence. Oxygen initiation and targets. The decision to initiate supplemental oxygen in adults with COVID-19 has not been studied, but indirect evidence from studies in pneumonia from another cause suggests that an oxygen saturation $\left(\mathrm{SpO}_{2}\right)$ less than $90 \%$ is associated with an increase in mortality and duration of hospitalization. ${ }^{1}$ Silent hypoxia is described as low oxygen levels with normal/hypercapnic respiratory failure and 
presenting without dyspnea. Potential mechanisms of mismatch between the severity of hypoxemia and the relatively mild respiratory discomfort may be a consequence of hypoxemia-driven hyperventilation and/or leftward shift of the oxyhemoglobin dissociation curve. This shift increases the alveolar oxygen tension and systemic saturation after reducing alveolar carbon dioxide tension, the Bohr effect. $\mathrm{PaO}_{2}$ and oxygen delivery can be optimized by modulating blood $\mathrm{pH}, \mathrm{PaCO}_{2}$, hemoglobin concentration, cardiac output, and arterial content of oxygen. These factors mean close attention is warranted when implementing lung-protective strategies, particularly when using low oxygen targets $(55-70 \mathrm{mmHg})$ and permissive hypercapnia. However, inappropriate use of supplementary oxygen in patients at risk of hypercapnic respiratory failure can result in life-threatening hypercapnia. ${ }^{2,3}$ Therefore, these patients need to be identified before prescribing and administering supplementary oxygen. Finally, a really important point to address is that frequently arterial blood gas analyses are not widely available as in HICs.

Ten randomized controlled trials (1,458 participants) reported an increase in the number of serious adverse events with higher fractions or oxygenation targets. ${ }^{4}$ One metaanalysis of 25 randomized clinical trials in critically ill patients before the COVID-19 pandemic shows that liberal oxygen therapy increases mortality without improving other patientimportant outcomes and that supplemental oxygen might result in harm when provided if $\mathrm{SpO}_{2}>97 \%{ }^{5}$ Recent randomized clinical trials showed no benefit or harm of a conservative oxygen therapy, when compared with a strategy that used usual oxygen targets, ${ }^{6}$ or a more liberal strategy. ${ }^{7}$

COVID-19 pneumonia may be more susceptible to potentially harmful effects of oxygen because adaptive responses are impaired by local inflammation and damage. ${ }^{8}$ In addition, COVID-19 patients tolerate hypoxemia pretty well, even when $\mathrm{SpO}_{2}$ decreases to $<88 \%$ probably because of a large intrapulmonary shunt with well-preserved lung compliance, which is unlike many other cases of ARDS by other causes. ${ }^{9}$ Targeting permissive hypoxemia $\left(\mathrm{PaO}_{2} 55-70 \%, \mathrm{SpO}_{2} 88-95 \%\right)$ in COVID-19 patients without pre-existing conditions that may make more severe hypoxemia unsafe is appealing (e.g., stroke, new myocardial infarction without revascularization, and neurotrauma). ${ }^{10}$ Of note, this approach may not be different from that practiced in HICs.

Oxygen delivery interfaces. A non-rebreather mask is a safe interface in supplemental oxygen therapy in COVID-19 patients as these masks help limit the dispersion of droplets. Human mannequin studies show that the maximum exhaled dispersion distance at $10 \mathrm{~L} /$ minute is $<10 \mathrm{~cm}$, suggesting that this modality generates the least dispersed aerosols. ${ }^{11}$ Nonrebreather masks can reach $\mathrm{FiO}_{2}$ of $>85 \%$ at flow rates approaching $15 \mathrm{~L} /$ minute. $^{12}$ To prevent hypercapnia, the reservoir bag must remain inflated at all times; this requires flow rates of at least $8-10 \mathrm{~L} /$ minute. $^{13}$ The use of nonrebreather masks usually requires oxygen via a piped system within the hospital.

High flow nasal oxygen, characterized as devices providing oxygen with flows from 20 to $70 \mathrm{~L} /$ minute, and NIV may reduce the need for intubation ${ }^{14,15}$ and could improve outcome in acute hypoxemic respiratory failure. ${ }^{16}$ In LMICs where the options for respiratory support can be severely limited, especially the first interface could be the best alternative. Several practical considerations need to be mentioned, though. First, patients should be closely monitored so that they can be intubated quickly when gas exchange worsens, or in case of increased work of breathing. ${ }^{17}$ Delayed intubation was associated with adverse outcomes and high failure rates in patients with the Middle East respiratory syndrome. ${ }^{18,19}$ Second, there may be more aerosol formation, ${ }^{20,21}$ which could increase the risk of viral transmission, although this may be limited when correct interfaces are used. ${ }^{22,23}$ Simulation studies suggest that surgical masks placed over the HFNO interface may reduce exhaled air dispersion (Figure 1). ${ }^{24,25}$ Also, during NIV, strict control of any air leak is recommended, and if a single limb circuit is used, a viral filter should be placed on the exhalation port of the mask (Figure 1). ${ }^{26}$ To date, no article comparing different interfaces for oxygen delivery in COVID19 in LMICs is available.

Fever control, use of cough suppressants in case of severe coughing, and light sedation. COVID-19 patients at times have high to very high minute volumes, which eventually leads to respiratory exhaustion and the need for endotracheal intubation. Because minute volume increases with body temperature, adequate and strict fever control could help preventing exhaustion, and thus, there is the need for invasive ventilation. One frequent symptom of COVID-19 is a persistent and severe cough, during which severe hypoxemia could develop. Cough control may be helpful, for example, by giving codeine. Light sedation may also reduce minute volume and also reduce coughing. In the absence of any literature on fever control, cough control, and light sedation in COVID-19 patients, this bundle, often combined with prone positioning, is currently practiced in non-intubated COVID-19 patients in the centers of the authors of this set of suggestions and recommendations.

Availability, affordability, safety, and feasibility. Improper use of supplementary oxygen occurs often in LMICs, frequently resulting in low pulse oximetry readings. ${ }^{27}$ As our appreciation of the risks of hypoxia, combined with the need to optimize the use of a scarce resource, we suggest starting supplementary oxygen when $\mathrm{SpO}_{2}$ is persistently lower than $94 \%$ for COVID19 patients.

In places where pulse oximetry is available, targeting a low $\mathrm{SpO}_{2}$ seems a safe strategy. Pulse oximeters are affordable and widely available and may be shared between COVID-19 patients in places with scarcity. Supplemental oxygen with lowest possible $\mathrm{FiO}_{2}$ helps prioritization of oxygen flow to the most severely ill patients within hospitals. However, in settings where continuous pulse oximetry is not available, it could be safer to use slightly higher targets for $\mathrm{SpO}_{2}\left(\mathrm{SpO}_{2}\right.$ at $\left.95-97 \%\right)$.

Low-flow supplemental oxygen via nasal prongs, a spongetipped catheter, or a face mask is widely available and affordable. ${ }^{10}$ High-flow systems need piped oxygen systems, which is often not available in LMIC hospitals. Oxygen concentrators may be a solution, but these are not widely available, expensive, and may be unsafe in settings with electricity fluctuations or power outages. High flow nasal oxygen and NIV are both feasible in LMICs, but come at additional costs for the machines and interfaces, have technical challenges and practical concerns, demand close monitoring, and depend on a stable oxygen supply. In settings with electricity fluctuations or blackouts, safety could improve when the devices used for HFNO and NIV have battery backup options. High flow nasal oxygen and NIV generate aerosols and should therefore be used in combination with a surgical mask or, preferably, in an 


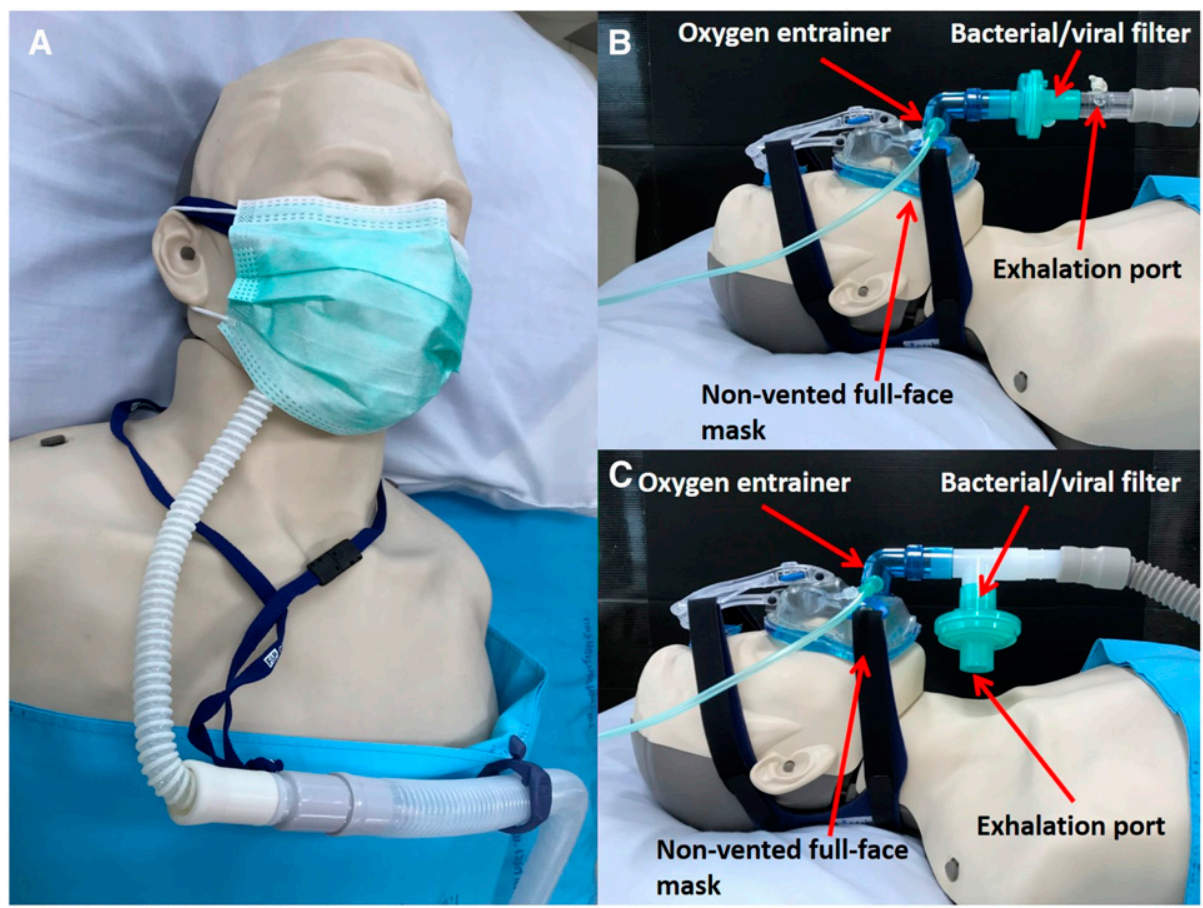

FIGURE 1. Oxygen delivery interfaces with HFNO and NIV in COVID-19 patients. (A) Placement of a surgical mask over the HFNO interface may reduce exhaled air dispersion. (B and $\mathbf{C})$ Example of single limb NIV circuit setup with a non-vented mask and viral filter. HFNO = high-flow nasal oxygen; NIV = noninvasive ventilation. Source: Produced by Chaisith Sivakorn, thanks to Napid Wadmanee, respiratory nurse at the Hospital for Tropical Diseases, Bangkok, Thailand, for the graphical input; permission is granted for the reuse of this figure.

isolation room. In addition, in settings where oxygen supply is limited, HFNO is not a good option as it has a high need for oxygen.

For fever control and cough suppression, paracetamol and codeine are widely available and cheap, and their use is without additional risk for patients. Nonsteroidal antiinflammatory drugs are suggested to be harmful, ${ }^{28-30}$ which could outweigh a potential benefit on symptom control for respiratory tract infection. ${ }^{31}$ For light sedation, benzodiazepines and morphine are widely available and cheap. Light sedation could be a challenge in resource-limited settings, although when there is lack of monitoring, training, or experience. Light sedation with small boluses of benzodiazepine, like midazolam, if needed combined with small boluses of morphine, is safe in experienced hands. It should be remembered that benzodiazepines could worsen or even induce agitation, or delirium. In those cases, it should be stopped. To improve tolerance to the NIV interface, or in case of delirium, dexmedetomidine, if available, is preferred over haloperidol because of significant QT interval prolongations. ${ }^{32-34}$

Recommendations and suggestions (Table 1).

1. In hypoxemic COVID-19 patients in LMICs, we suggest starting supplementary oxygen when $\mathrm{SpO}_{2}$ is persistently lower than $94 \%$ (weak recommendation, low quality of evidence).

2. In hypoxemic COVID-19 patients in LMICs, we recommend supplemental oxygen to keep $\mathrm{SpO}_{2}$ at $88-95 \%$ (strong recommendation, high quality of evidence).

3. In hypoxemic COVID-19 patients in LMICs, we suggest higher targets for $\mathrm{SpO}_{2}$ in settings where continuous pulse oximetry is not available $\left(\mathrm{SpO}_{2}\right.$ at $\left.95-97 \%\right)$ (weak recommendation, low quality of evidence).
4. In hypoxemic COVID-19 patients in LMICs, we recommend using low-flow oxygen systems, for example, nasal prongs, a sponge-tipped catheter, or a facial mask for supplementary oxygen, and, where available, a non-rebreathing mask (strong recommendation, low quality of evidence).

5. In COVID-19 patients in LMICs who remain hypoxemic despite use of low-flow oxygen therapy, we suggest the use of HFNO or NIV with monitoring and isolation considerations (especially for NIV), as discussed in the text (weak recommendation, low quality of evidence).

6. In COVID-19 patients in LMICs, we suggest fever control with paracetamol, using cough-suppressants, in case of severe coughing, and light sedation (weak recommendation, low quality of evidence).

Should prone positioning be used in COVID-19 in LMICs? Rationale. Prone positioning is associated with improvement in oxygenation and survival in patients with ARDS under invasive mechanical ventilation. The use of prone positioning before and after intubation is being discussed in patients with COVID-19 under oxygen therapy.

Search results. We searched the databases of PubMed and Embase through Ovid until November 1, 2020. The following terms were used, either as MeSH terms or as free text words: "COVID-19," "coronavirus," "novel corona," "SARS-CoV-2," "prone," and "prone positioning." No randomized clinical trial on the topic was found. All but one study was conducted in HICs.

Evidence. A multicenter study conducted in patients with acute respiratory failure and no COVID-19 reported prone positioning in non-intubated patients as a feasible and wellaccepted intervention that was associated with improvement 
in oxygenation. ${ }^{35}$ In another multicenter study in nonintubated moderate to severe ARDS patients under NIV or HFNO, it was suggested that early application of prone positioning with HFNO, especially in patients with moderate ARDS and baseline $\mathrm{SpO}_{2}>95 \%$, may help avoid intubation. ${ }^{36}$

Specifically in COVID-19 patients, in one study involving 24 patients, $63 \%$ of patients were able to tolerate prone positioning for more than 3 hours, but only $25 \%$ had an improvement in oxygenation Also, the study was not powered to assess clinical outcomes, but $21 \%$ of the patients required intubation within 10 days. ${ }^{37}$ Another case series of 15 COVID19 patients showed that the combination of prone positioning and NIV on general wards was feasible and resulted in a decrease in respiratory rates and increase in oxygenation. ${ }^{38}$ The only case series in an LMIC (Iran) was conducted in 10 patients and reported improvement in $\mathrm{SpO}_{2}$ and a decrease in dyspnea, with no intubation in the group. ${ }^{39}$ Additional case series confirmed the feasibility of the maneuver and its association with improvement in oxygenation and dyspnea. ${ }^{40-42}$ The impact of prone positioning in non-intubated patients on patientcentered outcomes remains unknown because no randomized clinical trial is still available.

In patients receiving mechanical ventilation, there is strong agreement to use prone positioning in severe cases of ARDS, ${ }^{43}$ which is supported by evidence from a randomized controlled trial. ${ }^{43-45}$ Based on our personal experience, we suggest a strategy of prone positioning in non-intubated patients receiving oxygen, HFNO, or NIV and without signs of severe respiratory distress. Patients should be kept in prone positioning as long as possible but aiming at least 3 hours per session. Of note, this approach may not be different from that practiced in HICs. In intubated patients with $\mathrm{PaO}_{2} / \mathrm{FiO}_{2}<$ $150 \mathrm{mmHg}$, prone positioning for 12-16 hours should be implemented.

Availability, affordability, safety, and feasibility. In places with an adequate number and trained staff, trying a strategy of prone positioning in patients with hypoxemia seems safe. Close monitoring, and a clear failure and escalation criteria are needed. The use of this strategy can help avoid some intubations and help the overall system at-risk of shortage of equipment. However, among the limitations of prone positioning is the workload for healthcare professionals; however, this is only a relative contraindication for prone positioning.

Recommendations and suggestions (Table 1).

1. In COVID-19 patients in LMICs who remain hypoxemic despite use of oxygen, HFNO or NIV and who do not exhibit clear signs of respiratory distress, we suggest a trial of prone positioning; this however requires close monitoring, and clear failure and escalation criteria (weak recommendation, low quality of evidence).

2. In intubated patients with COVID-19 in LMICs, we recommend using prone positioning for 12-16 hours in case of refractory hypoxemia $\left(\mathrm{PaO}_{2} / \mathrm{FiO}_{2}<150 \mathrm{mmHg}\right.$ ) (strong recommendation, low quality of evidence).

When to intubate for invasive ventilation in COVID-19 patients in LMICs? Rationale. The decision and timing of intubation is crucial in the management of patients with COVID19. The decision to intubate these patients, especially in LMICs, must weigh the risks of premature intubation against the risk of delayed intubation and its consequences, like respiratory arrest and an increase of risk of poor outcome.
Search results. We searched the databases of PubMed and Embase through Ovid until November 1, 2020. The following terms were used, either as MeSH terms or as free text words: "COVID-19," "coronavirus," "novel corona," "SARS-CoV-2," "intubation criteria," "hypoxemia," "silent hypoxemia," and "initiation of invasive ventilation."

Evidence. "Silent hypoxemia," characterized by severe desaturations with no or only some dyspnea, is frequently seen in COVID-19 patients. ${ }^{46,47}$ It seems that dyspnea in COVID-19 patients, if this develops, does not result from hypoxemia alone but is due to other causes, such as airflow obstruction, dead space ventilation, respiratory muscle dysfunction, and anxiety. ${ }^{46,48}$ Thus, hypoxemia alone is not necessarily an indication for intubation in COVID-19 patients. In addition, the respiratory rate is an important parameter to be followed up in these patients, and tachypnea is one of the signs that could suggest that intubation is needed. This concept was implemented by the Chinese Society of Anesthesiology, which recommends to proceed with endotracheal intubation in COVID-19 patients showing no improvement in respiratory distress or tachypnea (respiratory rate $>30$ / minute) in combination with worsening oxygenation $\left(\mathrm{PaO}_{2} /\right.$ $\mathrm{FiO}_{2}<150 \mathrm{mmHg}$ ) after applying 2-hour HFNO or NIV. ${ }^{47}$ This measure emphasizes more the worsening of respiratory symptoms, rather than focusing on hypoxemia per se. It is important to emphasize that with the evolving of the pandemic, a more liberal approach is being used, with sustained use of noninvasive support as long as the patient does not present signs of discomfort or clinical deterioration. In addition, the ROX, an index combining the respiratory rate and oxygenation (using the $\mathrm{SpO}_{2} / \mathrm{FiO}_{2}$ ) to predict outcome outperformed the diagnostic accuracy of the two variables separately, but was only validated in patients undergoing HFNO. The ROX index was also recently validated in COVID-19 patients, but again only in those receiving HFNO. Yet, the prognostic value in limited-resource settings and beyond the HFNO domain and potential diagnostic properties have to be proven. Whenever invasive ventilation is needed, it should be timely and effectively provided. Waiting to intubate hypoxemic patients until they require $\mathrm{FiO}_{2}$ of close to 1.0 may make intubation a challenge. Despite no formal evidence available for the best timing of intubation in COVID-19 patients, this recommendation is in line with previous guidelines of respiratory support of patients in LMICs. ${ }^{49,50}$

Availability, affordability, safety, and feasibility. Initiating invasive ventilation based on clinical signs like fatigue and exhaustion, in combination with refractory hypoxemia, helps prioritize intubation in COVID-19 patients to the ones who really need it. However, close monitoring for worsening signs of respiratory symptoms could be a challenge in LMICs, in particular when less experienced healthcare workers have to take care of these patients in the surge of patients who need hospitalization. ${ }^{51-53}$ When arterial blood gases are not available, the $\mathrm{PaO}_{2} / \mathrm{FiO}_{2}$ ratio could be replaced by the $\mathrm{SpO} / 2 / \mathrm{FiO}_{2}$ according to well-defined formulas. ${ }^{54-57}$ When available, intubation teams formed by skilled professionals and with predefined protocols for airway management should be considered.

Recommendations and suggestions (Table 1).

1. In hypoxemic COVID-19 patients in LMICs, we recommend to intubate and initiate invasive ventilation based on signs of fatigue and respiratory distress more than on refractory 
hypoxemia alone (weak recommendation, low quality of evidence).

2. In hypoxemic COVID-19 patients in LMICs, we recommend close monitoring for respiratory worsening and early intubation if worsening occurs (strong recommendation, low quality of evidence).

3. In hypoxemic COVID-19 patients in LMICs, we recommend staff members to be readily available to closely monitoring a patient when an early intubation was not performed, and staff should be trained in timely recognition of respiratory distress and sign of worsening of respiratory distress (weak recommendation, low quality of evidence).

How to set the ventilator in COVID-19 patients in LMICs? Rationale. Data to support evidence-based management of invasive ventilation in COVID-19 patients remain scarce. However, an adequate ventilatory strategy is frequently associated with better outcomes in patients receiving mechanical ventilation, and this should not be different in COVID-19 patients.

Search results. We searched the databases of PubMed and Embase through Ovid until November 1, 2020. The following terms were used, either as MeSH terms or as free text words: "COVID-19" and "mechanical ventilation,"

Evidence. All major studies assessing the mechanical ventilation strategy were conducted in HICs. The evidence-based principles underpinning invasive ventilation for patients with ARDS should also be considered for COVID-19 patients: provide ventilation to protect the lungs using low tidal volumes and keep airway pressures low (both plateau $\left[<30 \mathrm{cmH}_{2} \mathrm{O}\right]$ and driving pressures $\left[<15 \mathrm{cmH}_{2} \mathrm{O}\right]$ ). All articles that discussed ventilation strategies recommended the use of lowtidal volume ventilation with tidal volumes anywhere between 4 and $8 \mathrm{~mL} / \mathrm{kg}$ of predicted body weight (PBW) according to recommendation by the ARDS Network, derived from HICs. ${ }^{45,46,58-66}$ Among articles suggesting severe COVID-19 pneumonia phenotypes, at least one suggested using tidal volumes on the higher end of the spectrum (6-8 mL/kg PBW) to attenuate dyspnea. ${ }^{58}$ However, this recommendation is based on small case series of low quality and on phenotypes not externally validated. Thus, this strategy should not be considered as a standard of ventilation strategy for COVID-19 patients.

Among the studies that discussed phenotypes of patients with severe COVID-19 pneumonia requiring invasive mechanical ventilation, ${ }^{45,46,58-65}$ a common theme was the identification of two classes of patients: patients with a preserved to near normal ( $>50 \mathrm{~mL} / \mathrm{cmH}_{2} \mathrm{O}$ ) respiratory system compliance or those with a low respiratory systemic compliance $\left(<40 \mathrm{~mL} / \mathrm{cmH}_{2} \mathrm{O}\right)$. However, no validation or ventilation strategy tailored by these phenotypes was tested. At least five studies suggested using low to moderate levels of positive end-expiratory pressure (PEEP) $\left(8-10 \mathrm{cmH}_{2} \mathrm{O}\right)$ for those with preserved compliance and higher levels of PEEP $\left(>10 \mathrm{cmH}_{2} \mathrm{O}\right)$ among those with low compliance or a more typical presentation of ARDS. ${ }^{45,58-62}$ However, these recommendations were not validated in larger and well-performed multicenter studies or clinical trials. One study suggested overall poor alveolar recruitability in severe COVID-19 pneumonia. ${ }^{62}$

There was no specific recommendation on how to manage $\mathrm{FiO}_{2}$ relative to setting PEEP in any of the identified articles. One article discussed new evidence to aim for higher $\mathrm{PaO}_{2}$ or
$\mathrm{SpO}_{2}$ based on recent evidence compared with the recommendation of aiming for $\mathrm{PaO}_{2} 55-80 \mathrm{mmHg}$ or an $\mathrm{SpO}_{2} 88-92 \%$. ${ }^{46}$ There is no evidence that higher PEEP is more beneficial than lower PEEP regarding survival in patients with ARDS, with exception for a more severe group. ${ }^{67-69}$ In addition, there is no evidence that PEEP titrated according to the lower driving pressure results in better outcomes than the protocol-driven lower PEEP/ higher $\mathrm{FiO}_{2}$ tables. ${ }^{70}$ In fact, a recent randomized controlled trial of a strategy with lung recruitment and titrated PEEP compared with low PEEP increased 28-day all-cause mortality. ${ }^{71}$ Early experience with COVID-19 pneumonia is that many patients have remarkable mismatches between severity of hypoxemia and stiffness of the respiratory system. Indeed, static compliance is higher than what is usually found in ARDS from other etiologies. In such patients, high PEEP may result more in hyperinflation of normally aerated lung zones than in recruitment of collapsed regions.

In settings where there is limited availability of mechanical ventilators, unconventional approaches have been suggested, such as connecting multiple patients to a single ventilator. ${ }^{44,72-74}$ Specifically, when using parallel ventilation, tidal volumes could not be controlled and ranged between 257 and $621 \mathrm{~mL}$, even in patients with good compliance. ${ }^{72}$ Three identified articles cautioned against or did not endorse the practice but allowed room for consideration as a rescue strategy. ${ }^{73-75}$ When using invasive ventilation, there are clear recommendations about keeping the patient ventilator circuit as a closed system to avoid risk of aerosolization. Specifically, in one article, it was recommended clamping the endotracheal tube before any disconnection from the ventilator and placing the ventilator on standby before any circuit disconnection.

Availability, affordability, safety, and feasibility. Lack of mechanical ventilators poses a major management challenge in LMICs, especially during the COVID-19 pandemic, where anywhere between $2.3 \%$ and $33.1 \%$ of hospitalized patients may require mechanical ventilation. ${ }^{76}$ The number of ventilators per 100.000 inhabitants varies enormously in LMICs. In addition, in general, the outcomes of ventilated patients in LMICs, especially when the numbers of ventilators are low, are poor. This may be mainly related to safety issues. In settings where there is limited availability of mechanical ventilators, unconventional approaches have been suggested, such as connecting multiple patients to a single ventilator. ${ }^{72-74} \mathrm{How}-$ ever, there is a recommendation against this practice. ${ }^{72}$

Recommendations and suggestions (Table 1).

1. In intubated patients with COVID-19 in LMICs, we recommend using a low tidal volume (4-8 $\mathrm{mL} / \mathrm{kg} P B W)$, and whenever possible, a tidal volume $\leq 6 \mathrm{~mL} / \mathrm{kg}$ PBW should be pursued (strong recommendation, high quality of evidence).

2. In intubated patients with COVID-19 in LMICs, we recommend using a "low $\mathrm{PEEP} /$ high $\mathrm{FiO}_{2}$ table" (moderate recommendation, low quality of evidence).

3. In intubated patients with COVID-19 in LMICs, we recommend against using routine recruitment maneuvers, unless as a rescue therapy in refractory hypoxemia (moderate recommendation, low quality of evidence).

4. In intubated patients with COVID-19 in LMICs, we recommend against sharing one ventilator for multiple patients (strong recommendation, low quality of evidence).

How to wean a COVID-19 patient from the ventilator in LMICs? Rationale. The COVID-19 pandemic has resulted in 
the implementation of rapidly changing protocols and guidelines related to the indications and protocols for extubation. Both are aerosol-generating procedures, commonly associated with coughing, which necessitates proximity of the physician to the patient.

Search results. We searched the databases of PubMed and Embase through Ovid until November 01, 2020. The following terms were used, either as MeSH terms or as free text words: "COVID-19," "coronavirus," "novel corona," "SARS-CoV-2," "weaning," "spontaneous breathing trial," "tracheostomy," and "extubation." From the search, three randomized control trials, one systematic review, and four narrative reviews were found, and none of the studies were from LMICs. The search did not result in any studies from LMICs that directly answered the question of interest. We therefore discuss several pertinent studies from HICs.

Evidence. Weaning and spontaneous breathing trials. A meta-analysis of 10 randomized control trials including 3,165 patients showed no significant difference in the successful extubation, reintubation rate, ICU mortality, and ICU length of stay between spontaneous breathing trials (SBTs) using low levels of pressure support and a T-piece. ${ }^{77}$ Three wellperformed trials in resource-rich ICUs clearly showed benefit from early SBTs. ${ }^{78-80}$ However, because of much aerosol generating from a T-piece trial, a low-level pressure support is the preferred method for SBTs in COVID-19 patients. Anecdotal evidence demonstrated longer intubation periods, higher volume of secretions, and airway edema in mechanical ventilated COVID-19 patients, ${ }^{81}$ all of these factors increase the risk of post-extubation respiratory failure. Therefore, we suggest a higher degree of readiness for weaning and direct supplementation of low-flow oxygen after extubation in COVID-19 patients.

Extubation. The consensus statement published by an "Australian and New Zealand Intensive Care Society"7 and the "Difficult Airway Society, the Association of Anesthetists the Intensive Care Society, the Faculty of Intensive Care Medicine and the Royal College of Anesthetists,"82 and two additional opinions ${ }^{83,84}$ suggest that safe, simple, familiar, reliable, and robust practices should be adopted for all episodes of airway management for patients with COVID-19. Staff members should wear personal protective equipment (PPE) during extubation. Efforts should be made to minimize coughing and exposure to infected secretions at extubation time by previous suctioning of secretions. Before extubation, all necessary equipment should be checked. A regular surgical mask should then be placed on the patient's chin, and nasal prongs with low-flow oxygen should be applied after extubation. After extubation, ensure the patient immediately wears a face mask and their oxygen mask or nasal cannula, when practical. ${ }^{82}$ When available, a high-flow nasal cannula or NIV should be used after extubation to prevent reintubation. ${ }^{85}$ Information on tracheostomy is available in another article.

Availability, feasibility, affordability, and safety. Spontaneous breathing trials are available and affordable in LMICs. ${ }^{86}$ It is safer to perform SBTs using a low-level pressure support technique, rather than SBTs using a T-piece in COVID-19 patients; because with the former, a ventilatory support is guaranteed and little aerosol is generated. We recommend using the technique with low-level pressure support in all ventilated COVID-19 patients in resource-limited ICUs.
To reduce the risk of reintubation, we prefer a higher degree of extubation readiness in COVID-19 patients. This practice should include higher criteria for passing SBTs, or promote SBTs to last longer, or repeated for confirmation. Nurses and physicians should develop local SBT protocols, and oversedation should be recognized and avoided at the moment the decision is taken to proceed without invasive ventilation. Lowand middle-income countries have insufficient negative pressure airborne infectious isolation rooms in general. Then, at every extubation, one of the main aims should be the best possible protection of the healthcare workers. Personal protective equipment should be used and changed every time, and the extubating sequences should be safe, simple, familiar, and reliable according to available resources. A Canadian group restricted the aerosolization and droplet spraying during extubation with plastic drapes applied over the head and the endotracheal tube. ${ }^{87}$ Finally, whenever available, the physiotherapist should be involved in the weaning and extubation process.

Recommendations and suggestions (Table 1).

1. In COVID-19 patients in LMICs, we recommend daily assessments for readiness for weaning by a low-level pressure support (strong recommendation, low quality of evidence).

2. In COVID-19 patients in LMICs, we recommend against using a T-piece trial because of contamination risk (weak recommendation, low quality of evidence).

3. In COVID-19 patients in LMICs who are ready to extubate, we recommend using familiar, local extubation protocols to minimize infection risk among healthcare workers (weak recommendation, low quality of evidence).

Received July 3, 2020. Accepted for publication January 4, 2021.

Published online January 13, 2021.

Note: The Appendix can be found at www.ajtmh.org.

Financial support: Open access fees for this article and all others in this issue were supported by the Wellcome Trust of Great Britain.

Authors' addresses: Ary Serpa Neto, Australian and New Zealand Intensive Care Research Centre, Monash University, Melbourne, Australia, Department of Critical Care Medicine, Hospital Israelita Albert Einstein, São Paulo, Brazil, Department of Intensive Care, Amsterdam University Medical Centers, Location 'Academic Medical Center,' Amsterdam, The Netherlands, and Data Analytics Research and Evaluation (DARE) Centre, Austin Hospital, University of Melbourne, Melbourne, Australia, E-mail: ary.serpaneto@monash.edu. William Checkley, Division of Pulmonary and Critical Care, Department of Medicine, School of Medicine, Johns Hopkins University, Baltimore, MD, and Center for Global Non-Communicable Disease Research and Training, School of Medicine, Johns Hopkins University, Baltimore, MD, E-mail: wcheckl1@jhmi.edu. Chaisith Sivakorn, Department of Clinical Tropical Medicine, Mahidol University, Bangkok, Thailand, E-mail: chaisith.siv@mahidol.edu. Madiha Hashmi, Department of Anaesthesiology, Ziauddin University, Karachi, Pakistan, E-mail: madiha@nicslk.com. Alfred Papali, Division of Pulmonary and Critical Care Medicine, Atrium Health, Charlotte, NC, E-mail: Ifred.papali@atriumhealth.org. Marcus J. Schultz, Department of Intensive Care, Amsterdam University Medical Centers, Location 'Academic Medical Center,' Amsterdam, The Netherlands, Mahidol-Oxford Tropical Medicine Research Unit (MORU), Mahidol University, Bangkok, Thailand, and Nuffield Department of Medicine, Oxford University, Oxford, United Kingdom, E-mail: marcus.j.schultz@gmail.com.

This is an open-access article distributed under the terms of the Creative Commons Attribution (CC-BY) License, which permits unrestricted use, distribution, and reproduction in any medium, provided the original author and source are credited. 


\section{GROUP MEMBERS OF THE "COVID-LMIC TASK FORCE”}

Heads: Alfred Papali (Atrium Health, Charlotte, NC) and Marcus Schultz (Mahidol University, Bangkok, Thailand; University of Oxford, Oxford, United Kingdom; Amsterdam University Medical Centers, Location "AMC," Amsterdam, The Netherlands).

Advisors: Neill K. J. Adhikari (Interdepartmental Division of Critical Care Medicine, University of Toronto, Toronto, Canada); Emilie J. Calvello Hynes (University of Colorado School of Medicine, Department of Emergency Medicine, Denver, CO); Martin Dünser (Kepler University Hospital and Johannes Kepler University Linz, Linz, Austria) and Mervyn Mer (University of the Witwatersrand, Johannesburg, South Africa).

Subgroup members, in alphabetical order: Andrew Achilleos (Sunnybrook Health Sciences Centre, Toronto, Canada); Hanan Y. Ahmed (Department of Internal Medicine, College of Health Sciences, Addis Ababa University, Addis Ababa, Ethiopia); Kevan M. Akrami (Universidade Federal da Bahia, Faculdade de Medicina, Salvador, Brazil and Division of Infectious Disease, Department of Medicine, University of California San Diego, San Diego, CA); Lia M. Barros (University of Washington, Seattle, WA); B. Jason Brotherton (AIC Kijabe Hospital, Kijabe, Kenya); Sopheakmoniroth Chea (Calmette Hospital, Phnom Penh, Cambodia); William Checkley (The Johns Hopkins University School of Medicine and The Johns Hopkins University Center for Global Health, Baltimore, MD); Elif A. Cizmeci (Interdepartmental Division of Critical Care Medicine, Sunnybrook Health Sciences Centre, University of Toronto, Toronto, Canada); Natalie Cobb (University of Washington, Seattle, WA); Finot Debebe (Department of Emergency Medicine and Critical Care, Addis Ababa University School of Medicine, Addis Ababa, Ethiopia); Juliana Ferreira (University of São Paulo, São Paulo, Brasil); Gabriela Galli (University of Pittsburgh School of Medicine, Pittsburgh, PA); Tewodros Haile (Department of Internal Medicine, College of Health Sciences, Addis Ababa University); Bhakti Hansoti (Department of Emergency Medicine, Johns Hopkins University School of Medicine, Baltimore, MD); Madiha Hashmi (Ziauddin University, Karachi, Pakistan); Rebecca Inglis (Lao-Oxford-Mahosot Hospital-Wellcome Trust Research Unit [LOMWRU], Mahosot Hospital, Vientiane, Lao People's Democratic Republic and Department of Intensive Care, Oxford University Hospital Trust, Oxford, United Kingdom); Burton W. Lee (Critical Care Department, NIH, Bethesda, MD; Division of Pulmonary, Allergy and Critical Care, University of Pittsburgh School of Medicine, Pittsburgh, PA); Faith Lelei (AIC Kijabe Hospital, Kijabe, Kenya); Ganbold Lundeg (Mongolian National University of Medical Sciences, Ulan Bator, Mongolia); David Misango (The Aga Khan University, Nairobi, Kenya); Ary Serpa Neto (Department of Critical Care Medicine, Hospital Israelita Albert Einstein, São Paulo, Brazil; Australian and New Zealand Intensive Care Research Centre (ANZIC-RC), Monash University, Melbourne, Australia; Department of Intensive Care, Amsterdam University Medical Centers, Location "AMC," University of Amsterdam, Amsterdam, The Netherlands); Alfred Papali (Atrium Health, Charlotte, NC); Casey Park (Interdepartmental Division of Critical Care Medicine, Sunnybrook Health Sciences Centre, University of Toronto, Toronto, Canada); Rajyabardhan Pattnaik (Ispat General Hospital, Rourkela, India); Jennifer L. Pigoga (Division of Emergency Medicine, University of South Africa, Cape Town, South Africa); Luigi Pisani (Department of Anesthesia and Intensive Care, Miulli Regional Hospital, Acquaviva delle Fonti, Italy; Doctors with Africa - CUAMM, Padova, Italy; Mahidol-Oxford Tropical Medicine Research Unit (MORU), Mahidol University, Bangkok, Thailand); Elisabeth D. Riviello (Division of Pulmonary, Critical Care, and Sleep Medicine, Beth Israel Deaconess Medical Center and Harvard Medical School, Boston, MA); Kristina E. Rudd (University of Pittsburgh School of Medicine, Pittsburgh, PA); Marcus Schultz (Mahidol University, Bangkok, Thailand; University of Oxford, Oxford, United Kingdom; Amsterdam University Medical Centers, Location "AMC," Amsterdam, The Netherlands); Varun U. Shetty (University of Pittsburgh Medical Center, Pittsburgh, PA); Gentle S. Shrestha (Tribhuvan University Teaching Hospital, Kathmandu, Nepal); Chaisith Sivakorn (Mahidol University, Salaya, Thailand); Shaurya Taran (Interdepartmental Division of Critical Care Medicine, Sunnybrook
Health Sciences Centre, University of Toronto, Toronto, Canada); T. Eoin West (University of Washington, Seattle, WA).

Group members of the subgroup "Acute Respiratory Failure and Mechanical Ventilation": Ary Serpa Neto (Department of Critical Care Medicine, Hospital Israelita Albert Einstein, São Paulo, Brazil; Australian and New Zealand Intensive Care Research Centre (ANZIC-RC), Monash University, Melbourne, Australia; Department of Intensive Care, Amsterdam University Medical Centers, Location "AMC," University of Amsterdam, Amsterdam, The Netherlands), Marcus Schultz (Mahidol University, Bangkok, Thailand; University of Oxford, Oxford, United Kingdom; Amsterdam University Medical Centers, Location "AMC' "Amsterdam, The Netherlands), William Checkley (The Johns Hopkins University School of Medicine and The Johns Hopkins University Center for Global Health, Baltimore, MD), Chaisith Sivakorn (Mahidol University, Salaya, Thailand), Madiha Hashmi (Ziauddin University, Karachi, Pakistan), and Alfred Papali (Atrium Health, Charlotte, NC).

\section{REFERENCES}

1. Majumdar SR, Eurich DT, Gamble JM, Senthilselvan A, Marrie TJ, 2011. Oxygen saturations less than $92 \%$ are associated with major adverse events in outpatients with pneumonia: a population-based cohort study. Clin Infect Dis 52: 325-331.

2. O'Driscoll BR, Howard LS, Earis J, Mak V, 2017. British thoracic society guideline for oxygen use in adults in healthcare and emergency settings. BMJ Open Resp Res 4: e000170.

3. Roberts CM, Stone RA, Buckingham RJ, Pursey NA, Lowe D; National Chronic Obstructive Pulmonary Disease Resources and Outcomes Project Implementation Group, 2011. Acidosis, non-invasive ventilation and mortality in hospitalized COPD exacerbations. Thorax 66: 43-48.

4. Barbateskovic M, Schjørring OL, Krauss SR, Jakobsen JC, Meyhoff CS, Dahl RM, Rasmussen BS, Perner A, Wetterslev J, 2019. Higher versus lower fraction of inspired oxygen or targets of arterial oxygenation for adults admitted to the intensive care unit. Cochrane Database Syst Rev 2019: CD012631.

5. Chu DK, Kim LH, Young PJ, Zamiri N, Almenawer SA, Jaeschke R, Szczeklik W, Schünemann HJ, Neary JD, Alhazzani W, 2018. Mortality and morbidity in acutely ill adults treated with liberal versus conservative oxygen therapy (IOTA): a systematic review and meta-analysis. Lancet 391: 1693-1705.

6. ICU-ROX Investigators and the Australian and New Zealand Intensive Care Society Clinical Trials Group, 2020. Conservative oxygen therapy during mechanical ventilation in the ICU. NEngl J Med 12: 989-998.

7. Barrot $L$ et al., 2020. Liberal or conservative oxygen therapy for acute respiratory distress syndrome. NEngl J Med 12: 999-1008.

8. Griffith DE, Garcia JG, James HL, Callahan KS, Iriana S, Holiday D, 1992. Hyperoxic exposure in humans: effects of 50 percent oxygen on alveolar macrophage leukotriene B4 synthesis. Chest 101: 392-397.

9. Archer SL, Sharp WW, Weir EK, 2020. Differentiating COVID-19 pneumonia from acute respiratory distress syndrome (ARDS) and high-altitude pulmonary edema (HAPE): therapeutic implications. Circulation 142: 101-104.

10. Rassool RP, Sobott BA, Peake DJ, Mutetire BS, Moschovis PP, Black JF, 2017. A low-pressure oxygen storage system for oxygen supply in low-resource settings. Respir Care 62: 1582-1587.

11. Hui DS, Chan MT, Chow B, 2014. Aerosol dispersion during various respiratory therapies: a risk assessment model of nosocomial infection to health care workers. Hong Kong Med J 20 (Supp/ 4): 9-13.

12. Farias E, Rudski L, Zidulka A, 1991. Delivery of high inspired oxygen by face mask. J Crit Care 6: 119-124.

13. Herren T, Achermann E, Hegi T, Reber A, Staubli M, 2017. Carbon dioxide narcosis due to inappropriate oxygen delivery: a case report. J Med Case Rep 11: 204

14. Xu XP et al., 2017. Noninvasive ventilation in acute hypoxemic nonhypercapnic respiratory failure: a systematic review and meta-analysis. Crit Care Med 45: e727-e733.

15. Zhao H, Wang H, Sun F, Lyu S, An Y, 2017. High-flow nasal cannula oxygen therapy is superior to conventional oxygen therapy but 
not to noninvasive mechanical ventilation on intubation rate: a systematic review and meta-analysis. Crit Care 21: 184.

16. Frat JP et al., 2015. High-flow oxygen through nasal cannula in acute hypoxemic respiratory failure. $N$ Engl J Med 372: 2185-2196.

17. Kang BJ et al., 2015. Failure of high-flow nasal cannula therapy may delay intubation and increase mortality. Intensive Care Med 41: 623-632.

18. Alraddadi BM et al., 2019. Noninvasive ventilation in critically ill patients with the Middle East respiratory syndrome. Influenza Other Respir Viruses 13: 382-390.

19. Arabi YM, Arifi AA, Balkhy HH, Najm H, Aldawood AS, Ghabashi A, Hawa H, Alothman A, Khaldi A, Al Raiy B, 2014. Clinical course and outcomes of critically ill patients with Middle East respiratory syndrome coronavirus infection. Ann Intern Med 160: 389-397.

20. Tran K, Cimon K, Severn M, Pessoa-Silva CL, Conly J, 2012. Aerosol generating procedures and risk of transmission of acute respiratory infections to healthcare workers: a systematic review. PLoS one 7: e35797.

21. Ferioli M, Cisternino C, Leo V, Pisani L, Palange P, Nava S, 2020. Protecting healthcare workers from SARS-CoV-2 infection: practical indications. Eur Respir Rev 29: 200068.

22. de Jonge $E$, Peelen L, Keijzers PJ, Joore H, de Lange $D$, van der Voort PH, Bosman RJ, de Waal RA, Wesselink R, de Keizer NF, 2008. Association between administered oxygen, arterial partial oxygen pressure and mortality in mechanically ventilated intensive care unit patients. Crit Care 12: R156.

23. Kilgannon JH, Jones AE, Shapiro NI, Angelos MG, Milcarek B, Hunter K, Parrillo JE, Trzeciak S; Emergency Medicine Shock Research Network Investigators, 2010. Association between arterial hyperoxia following resuscitation from cardiac arrest and in-hospital mortality. JAMA 303: 2165-2171.

24. Li J, Fink JB, Ehrmann S, 2020. High-flow nasal cannula for COVID-19 patients: low risk of bio-aerosol dispersion. EurRespir J 55: 2000892.

25. Hui DS, Chow BK, Chu L, Ng SS, Lee N, Gin T, Chan MT, 2012. Exhaled air dispersion during coughing with and without wearing a surgical or N95 mask. PLoS one 7: e50845.

26. Durie M, 2020. NHS Guidance on Use of NIV in Adults with Coronavirus. Available at: https://www.england.nhs.uk/coronavirus/ secondary-care/other-resources/specialty-guides/. Accessed June 16, 2020.

27. Ginsburg AS, Van Cleve W, Thompson M, English M, 2012. Oxygen and pulse oximetry in childhood pneumonia: a survey of healthcare providers in re-source-limited settings. J Trop Pediatr 58: 389-393.

28. Basille D, Plouvier N, Trouve C, Duhaut P, Andrejak C, Jounieaux $V, 2017$. Non-steroidal anti-inflammatory drugs may worsen the course of community-acquired pneumonia: a cohort study. Lung 195: 201-208.

29. Legras A et al., 2009. A multicentre case-control study of nonsteroidal anti-inflammatory drugs as a risk factor for severe sepsis and septic shock. Crit Care 13: R43.

30. Messika J, Sztrymf B, Bertrand F, Billard-Pomares T, Barnaud G, Branger C, Dreyfuss D, Ricard JD, 2014. Risks of nonsteroidal antiinflammatory drugs in undiagnosed intensive care unit pneumococcal pneumonia: younger and more severely affected patients. J Crit Care 29: 733-738.

31. Kim SY, Chang YJ, Cho HM, Hwang YW, Moon YS, 2013. Nonsteroidal anti-inflammatory drugs for the common cold. Cochrane Database Syst Rev 6: CD006362.

32. Abdelgalel EF, 2016. Dexmedetomidine versus haloperidol for prevention of delirium during non-invasive mechanical ventilation. Egypt $J$ Anaesth 32: 473-481.

33. Reade MC, O'Sullivan K, Bates S, Goldsmith D, Ainslie WR, Bellomo R, 2009. Dexmedetomidine vs. haloperidol in delirious, agitated, intubated patients: a randomised open-label trial. Crit Care 13: R75.

34. Hanidziar D, Bittner EA, 2020. Sedation of mechanically ventilated COVID-19 patients: challenges and special considerations. Anesth Analg 131: e40-e41.

35. Scaravilli V, Grasselli G, Castagna L, Zanella A, Isgrò S, Lucchini A, Patroniti N, Bellani G, Pesenti A, 2015. Prone positioning improves oxygenation in spontaneously breathing nonintubated patients with hypoxemic acute respiratory failure: a retrospective study. J Crit Care 30: 1390-1394.
36. Ding L, Wang L, Ma W, He H, 2020. Efficacy and safety of early prone positioning combined with HFNC or NIV in moderate to severe ARDS: a multi-center prospective cohort study. Crit Care 24: 28.

37. Elharrar X, Trigui Y, Dols AM, Touchon F, Martinez S, Prud'homme E, Papazian L, 2020. Use of prone positioning in nonintubated patients with COVID-19 and hypoxemic acute respiratory failure. JAMA 323: 2336-2338.

38. Sartini C, Tresoldi M, Scarpellini P, Tettamanti A, Carcò F, Landoni G, Zangrillo A, 2020. Respiratory parameters in patients with COVID-19 after using noninvasive ventilation in the prone position outside the intensive care unit. JAMA 323: 2338-2340.

39. Moghadam VD, Shafiee H, Ghorbani M, Heidarifar R, 2020. Prone positioning in management of COVID-19 hospitalized patients. Braz J Anesthesiol 70: 188-190.

40. Ng Z, Tay WC, Ho CHB, 2020. Awake prone positioning for nonintubated oxygen dependent COVID-19 pneumonia patients. Eur Respir J 56: 2001198

41. Despres C, Brunin Y, Berthier F, Pili-Floury S, Besch G, 2020. Prone positioning combined with high-flow nasal or conventional oxygen therapy in severe COVID-19 patients. Crit Care 24: 256.

42. Xu Q, Wang T, Qin X, Jie Y, Zha L, Lu W, 2020. Early awake prone position combined with high-flow nasal oxygen therapy in severe COVID-19: a case series. Crit Care 24: 250.

43. Guérin $C$ et al., 2013. Prone positioning in severe acute respiratory distress syndrome. N Engl J Med 368: 2159-2168.

44. Robba $C$ et al., 2020. Distinct phenotypes require distinct respiratory management strategies in severe COVID-19. Respir Physiol Neurobiol 279: 103455.

45. Wilcox SR, 2020. Management of respiratory failure due to COVID-19. BMJ 369: $\mathrm{m} 1786$.

46. Tobin MJ, 2020. Basing respiratory management of coronavirus on physiological principles. Am J Respir Crit Care Med 201: 1319-1320.

47. Xie J, Tong Z, Guan X, Du B, Qiu H, Slutsky AS, 2020. Critical care crisis and some recommendations during the COVID-19 epidemic in China. Intensive Care Med 46: 837-840.

48. Baldwin J, Cox J, 2016. Treating dyspnea: is oxygen therapy the best option for all patients? Med Clin 100: 1123-1130.

49. Zuo MZ, Huang YG, Ma WH, Xue ZG, Zhang JQ, Gong YH, Che L; Chinese Society of Anesthesiology Task Force on Airway Management, 2020. Expert recommendations for tracheal intubation in critically ill patients with noval coronavirus disease 2019. Chin Med Sci J 35: 105-109.

50. Serpa Neto A, Schultz MJ, Festic E, 2016. Ventilatory support of patients with sepsis or septic shock in resource-limited settings. Intensive Care Med 42: 100-103.

51. Dondorp AM, Hayat M, Aryal D, Beane A, Schultz MJ, 2020. Respiratory support in COVID-19 patients, with a focus on resource-limited settings. Am J Trop Med Hyg 102: 1191-1197.

52. Bambi S, lozzo P, Lucchini A, 2020. New issues in nursing management during the COVID-19 pandemic in Italy. Am J Crit Care 29: e92-e93.

53. Hvarfner A, Blixt J, Schell CO, Castegren M, Lugazia ER, Mulungu M, Litorp H, Baker T, 2020. Vital signs directed therapy for the critically ill: improved adherence to the treatment protocol two years after implementation in an intensive care unit in Tanzania. Emerg Med Int 2020: 4819805.

54. Baker T, Schell CO, Lugazia E, Blixt J, Mulungu M, Castegren M, Eriksen J, Konrad D, 2015. Vital signs directed therapy: improving care in an intensive care unit in a low-income country. PLoS One 10: e0144801.

55. Pandharipande PP, Shintani AK, Hagerman HE, St Jacques PJ, Rice TW, Sanders NW, Ware LB, Bernard GR, Ely EW, 2009. Derivation and validation of $\mathrm{SpO} 2 / \mathrm{FiO} 2$ ratio to impute for $\mathrm{PaO} 2 / \mathrm{FiO} 2$ ratio in the respiratory component of the sequential organ failure assessment (SOFA) score. Crit Care Med 37: 1317-1321.

56. Riviello ED, Kiviri W, Twagirumugabe T, Mueller A, BannerGoodspeed VM, Officer L, Novack V, Mutumwinka M, Talmor DS, Fowler RA, 2016. Hospital incidence and outcomes of the acute respiratory distress syndrome using the Kigali modification of the Berlin definition. Am J Respir Crit Care Med 193: 52-59. 
57. Vercesi $V$ et al., 2018. External confirmation and exploration of the Kigali modification for diagnosing moderate or severe ARDS. Intensive Care Med 44: 523-524.

58. Li X, Ma X, 2020. Acute respiratory failure in COVID-19: is it "typical" ARDS? Crit Care 24: 198.

59. Gattinoni L, Chiumello D, Rossi S, 2020. COVID-19 pneumonia: ARDS or not? Crit Care 24: 154.

60. Gattinoni L, Coppola S, Cressoni M, Busana M, Rossi S, Chiumello D, 2020. COVID-19 does not lead to a "typical" acute respiratory distress syndrome. Am J Respir Crit Care Med 201: 1299-1300.

61. Roesthuis $L$, van den Berg M, van der Hoeven $H, 2020$. Advanced respiratory monitoring in COVID-19 patients: use less PEEP! Crit Care 24: 230

62. Pan C, Chen L, Lu C, Zhang W, Xia JA, Sklar MC, Du B, Brochard L, Qiu H, 2020. Lung recruitability in COVID-19-associated acute respiratory distress syndrome: a single-center observational study. Am J Respir Crit Care Med 201: 1294-1297.

63. Arabi YM, Fowler R, Hayden FG, 2020. Critical care management of adults with community-acquired severe respiratory viral infection. Intensive Care Med 46: 315-328.

64. Meng L et al., 2020. Intubation and ventilation amid the COVID-19 outbreak: Wuhan's experience. Anesthesiology 132: 1317-1332.

65. Higny J, Feye F, Forêt F, 2020. COVID-19 pandemic: overview of protective-ventilation strategy in ARDS patients. Acta Clin Belg $1-3$.

66. Acute Respiratory Distress Syndrome Network, 2020. Ventilation with lower tidal volumes as compared with traditional tidal volumes for acute lung injury and the acute respiratory distress syndrome. N Engl J Med 342: 1301-1308.

67. Barrot L, Asfar P, Mauny F; LOCO2 Investigators and REVA Research Network, 2020. Liberal or conservative oxygen therapy for acute respiratory distress syndrome. $N$ Engl J Med 382: 999-1008.

68. Briel $\mathrm{M}$ et al., 2010. Higher vs lower positive end-expiratory pressure in patients with acute lung injury and acute respiratory distress syndrome: systematic review and meta-analysis. JAMA 303: 865-873.

69. Fan E et al., 2017. An official American thoracic society/European society of intensive care medicine/society of critical care medicine clinical practice guideline: mechanical ventilation in adult patients with acute respiratory distress syndrome. $\mathrm{Am} \mathrm{J}$ Respir Crit Care Med 195: 1253-1263.

70. Sahetya SK, Goligher EC, Brower RG, 2017. Fifty years of research in ARDS. Setting positive end-expiratory pressure in acute respiratory distress syndrome. Am J Respir Crit Care Med 195: 1429-1438.

71. Writing Group for the Alveolar Recruitment for Acute Respiratory Distress Syndrome Trial (ART) Investigators, 2017. Effect of lung recruitment and titrated positive end-expiratory pressure (PEEP) vs low PEEP on mortality in patients with acute respiratory distress syndrome: a randomized clinical trial. JAMA 318: 1335-1345.

72. Lotz C, Notz Q, Kranke P, Kredel M, Meybohm P, 2020. Unconventional approaches to mechanical ventilation-step-bystep through the COVID-19 crisis. Crit Care 24: 233.

73. Tonetti T, Zanella A, Pizzilli G, Babcock Cl, Venturi S, Nava S, Pesenti A, Ranieri VM, 2020. One ventilator for two patients: feasibility and considerations of a last resort solution in case of equipment shortage. Thorax 75: 517-519.

74. Pearson SD, Hall JB, Parker WF, 2020. Two for one with split- or co-ventilation at the peak of the COVID-19 tsunami: is there any role for communal care when the resources for personalised medicine are exhausted? Thorax 275: 444-445.

75. Herrmann J, da Cruz AF, Hawley ML, Branson RD, Kaczka DW, 2020. Shared ventilation in the era of COVID-19: a theoretical consideration of the dangers and potential solutions. Respir Care 65: 932-945.

76. Wunsch $\mathrm{H}, 2020$. Mechanical ventilation in COVID-19: interpreting the current epidemiology. Am J Respir Crit Care Med 202: 1-4.

77. Li Y, Li H, Zhang D, 2020. Comparison of T-piece and pressure support ventilation as spontaneous breathing trials in critically ill patients: a systematic review and meta-analysis. Crit Care 24: 67.

78. Ely EW, Baker AM, Dunagan DP, Burke HL, Smith AC, Kelly PT, Johnson MM, Browder RW, Bowton DL, Haponik EF, 1996.
Effect on the duration of mechanical ventilation of identifying patients capable of breathing spontaneously. $N$ Engl $J$ Med 335: 1864-1869.

79. Girard TD et al., 2008. Efficacy and safety of a paired sedation and ventilator weaning protocol for mechanically ventilated patients in intensive care (awakening and breathing controlled trial): a randomised controlled trial. Lancet 371 : 126-134.

80. Kress JP, Pohlman AS, O'Connor MF, Hall JB, 2000. Daily interruption of sedative infusions in critically ill patients undergoing mechanical ventilation. N Engl J Med 342: 1471-1477.

81. Grasselli $\mathrm{G}$ et al., 2020. Baseline characteristics and outcomes of 1591 patients infected with SARS-CoV-2 admitted to ICUs of the Lombardy region, Italy. JAMA 323: 1574-1581.

82. Cook TM, El-Boghdadly K, McGuire B, McNarry AF, Patel A, Higgs $A, 2020$. Consensus guidelines for managing the airway in patients with COVID-19: guidelines from the difficult airway society, the association of anaesthetists the intensive care society, the faculty of intensive care medicine and the royal college of anaesthetists. Anaesthesia 75: 785-799.

83. D'Silva DF, McCulloch TJ, Lim JS, Smith SS, Carayannis D, 2020. Extubation of patients with COVID-19. Br J Anaesth 125: e192-e195.

84. Asenjo JF, 2020. Safer intubation and extubation of patients with COVID-19. Can J Anaesth 67: 1276-1278.

85. Granton D et al., 2020. High-flow nasal cannula compared with conventional oxygen therapy or noninvasive ventilation immediately postextubation: a systematic review and meta-analysis. Crit Care Med 48: e1129-e1136.

86. Dondorp AM, Dünser MW, Schultz MJ, ed., 2019. Sepsis Management in Resource-Limited Settings. Switzerland: Springer.

87. Matava CT, Yu J, Denning S, 2020. Clear plastic drapes may be effective at limiting aerosolization and droplet spray during extubation: implications for COVID-19. Can J Anaesth 67: 902-904.

\section{APPENDIX}

\section{Development of recommendations and suggestions.}

Selection of Task Force members. The selection of the group members was based on interest in specific aspects of COVID19 and direct experience in low- and middle-income countries (LMICs. Alfred Papali and Marcus Schultz contacted potential team members through email and in person early in the pandemic of COVID-19 and created 8 subgroups assigned to separate areas in COVID-19 management, that is, "triage," "safety," "organization," "microbiology and laboratory tests, imaging tools, and diagnostic and prognostic modeling," "acute respiratory failure," "acute kidney injury," "coagulopathy," "prevention and therapy," "shock," and "support after initial care." In total, there were 38 Task Force members representing five medical specialties or disciplines (emergency medicine, intensive care, infectious diseases, internal medicine, and critical care nursing) from five of six WHO geographic regions. The Task Force consisted of 16 full-time LMIC members, 16 full-time HIC members-all with direct LMIC experience-and six members with joint LMIC/HIC appointments.

Selection of subgroup members. Ary Serpa Neto, William Checkley, Chaisith Sivakorn, Madiha Hashmi, Marcus Schultz, and Alfred Papali were assigned to this subgroup based on their specific expertise and interest in acute respiratory failure and mechanical ventilation.

Meetings. An initial Internet subgroup heads meeting was held to establish the procedures for literature review and drafting tables for evidence analysis. The subgroup heads continued work via the Internet. Several meetings occurred through teleconferences and electronic-based discussions among the subgroup heads and with members of other subgroups.

In the first meetings, a set of clearly defined questions regarding laboratory tests, imaging tools, and diagnostic and 
TABLE A1

Quality of evidence

\begin{tabular}{lll}
\hline A & Randomized clinical trials & High \\
B & $\begin{array}{l}\text { Downgraded randomized clinical trial(s) or } \\
\text { upgraded observational studies }\end{array}$ & High \\
C & $\begin{array}{l}\text { Observational studies } \\
\text { Downgraded observational studies or } \\
\text { expert opinions }\end{array}$ & Low \\
& Low
\end{tabular}

Factors that may decrease strength of evidence include high likelihood of bias; inconsistency of results, including problems with subgroup analyses; indirectness of evidence (other population, intervention, control, outcomes, and comparison); imprecision of findings; and likelihood of reporting bias.

Factors that may increase strength of evidence: large magnitude of effect (direct evidence, relative risk $>2$ with no plausible confounders); very large magnitude of effect with relative risk $>5$ and no threats to validity (by two levels); and dose-response gradient.

Adapted from Dondorp AM, Dünser MW, Schultz MJ, eds., 2019. Sepsis Management in Resource-limited Settings. Springer. doi.org/10.1007/978-3-030-03143-5.

prognostic modeling were formulated. These were reviewed for content and clarity by the subgroup members and heads from the other subgroups. After approval by the subgroup members and heads from the other subgroups, the subgroup members split up, each seeking evidence for recommendations regarding three or four of the specific questions posed, seeking help from the subgroup members in identifying relevant publications, where necessary. During this process, questions could be combined, so the subgroup heads were finally left with four major questions. The subgroup heads summarized the evidence in the Appendix and formulated a set of recommendations and suggestions after online discussions. These were communicated among the subgroup members. After their approval, the subgroup heads summarized the evidence in a report, which was sent for approval by all members of the Task Force.

Search techniques. The literature search followed the same techniques as previously described. ${ }^{1}$ In case a question was identical to one in those recommendations, the subgroup members only searched for additional articles, specifically new investigations or meta-analyses related to the questions, in a minimum of one general database (i.e., MEDLINE and Embase) and the Cochrane libraries. Furthermore, the subgroup members identified investigations from LMICs and also searched for unpublished study results.

Grading of Recommendations. The subgroup members classified quality of evidence as high or low and recommendations as strong or weak. The factors influencing this classification are presented in Table A1.
TABLE A2

Strong vs. weak recommendations*

\begin{tabular}{|c|c|}
\hline What is considered & How it affects the recommendation? \\
\hline High evidence & $\begin{array}{l}\text { The higher the quality of evidence, } \\
\text { the more likely a strong } \\
\text { recommendation }\end{array}$ \\
\hline $\begin{array}{l}\text { Certainty about the balance of } \\
\text { benefits vs. harms and burdens }\end{array}$ & $\begin{array}{l}\text { The larger/smaller the difference } \\
\text { between the desirable and } \\
\text { undesirable consequences and } \\
\text { the certainty around that } \\
\text { difference, the more likely a } \\
\text { strong/weak recommendation }\end{array}$ \\
\hline Certainty in or similar values & $\begin{array}{l}\text { The more certainty or similarity in } \\
\text { values and preferences, the } \\
\text { more likely a strong } \\
\text { recommendation }\end{array}$ \\
\hline Resource implications & $\begin{array}{l}\text { The lower/higher the cost of an } \\
\text { intervention compared to the } \\
\text { alternative the more likely a } \\
\text { strong/weak recommendation }\end{array}$ \\
\hline $\begin{array}{l}\text { Availability and feasibility in } \\
\text { LMICs }\end{array}$ & $\begin{array}{l}\text { The less available, the more likely } \\
\text { a weak recommendation }\end{array}$ \\
\hline Affordability for LMICs & $\begin{array}{l}\text { The less affordable, the more } \\
\text { likely a weak recommendation }\end{array}$ \\
\hline Safety of the intervention in LMICs & $\begin{array}{l}\text { The less safe in an LMIC, the more } \\
\text { likely a weak recommendation }\end{array}$ \\
\hline
\end{tabular}

The subgroup members paid extensive attention to several other factors as used before, but now focusing on LMICs, that is, availability and feasibility in LMICs, and safety matters in LMICs. A strong recommendation was worded as "we recommend" and a weak recommendation as "we suggest." A number of recommendations could remain "ungraded,", when, in the opinion of the subgroup members, such recommendations were not conducive for the process described above (Table A2).

Reporting. The report was edited for style and form by Alfred Papali or Marcus Schultz, with final approval by subgroup heads and then by the entire "COVID-LMIC Task Force." A final document was submitted to the "American Journal of Tropical Medicine and Hygiene" for potential publication as a 1,000-word article and made open access.

Financial support. Open access fees for this manuscript, and all nine others in the series, were supported by the Wellcome Trust of Great Britain. 